BNL-77528-2007-CP

\title{
Study of electron-proton beam-beam interaction in eRHIC
}

\author{
Y. Hao, V.N. Litvinenko, C. Montag, E. Pozdeyev, V. Ptitsyn
}

Presented at the $22^{\text {nd }}$ Particle Accelerator Conference (PAC)

Albuquerque, New Mexico

June $25-29,2007$

\author{
Collider-Accelerator Department \\ Brookhaven National Laboratory \\ P.O. Box 5000 \\ Upton, NY 11973-5000 \\ www.bnl.gov
}

Notice: This manuscript has been authored by employees of Brookhaven Science Associates, LLC under Contract No. DE-AC02-98CH10886 with the U.S. Department of Energy. The publisher by accepting the manuscript for publication acknowledges that the United States Government retains a non-exclusive, paid-up, irrevocable, world-wide license to publish or reproduce the published form of this manuscript, or allow others to do so, for United States Government purposes.

This preprint is intended for publication in a journal or proceedings. Since changes may be made before publication, it may not be cited or reproduced without the author's permission. 


\section{DISCLAIMER}

This report was prepared as an account of work sponsored by an agency of the United States Government. Neither the United States Government nor any agency thereof, nor any of their employees, nor any of their contractors, subcontractors, or their employees, makes any warranty, express or implied, or assumes any legal liability or responsibility for the accuracy, completeness, or any third party's use or the results of such use of any information, apparatus, product, or process disclosed, or represents that its use would not infringe privately owned rights. Reference herein to any specific commercial product, process, or service by trade name, trademark, manufacturer, or otherwise, does not necessarily constitute or imply its endorsement, recommendation, or favoring by the United States Government or any agency thereof or its contractors or subcontractors. The views and opinions of authors expressed herein do not necessarily state or reflect those of the United States Government or any agency thereof. 


\title{
STUDY OF ELECTRON-PROTON BEAM-BEAM INTERACTION IN ERHIC*
}

\author{
Y. Hao", V.N. Litvinenko, C. Montag, E. Pozdeyev, V. Ptitsyn, BNL, Upton, NY 11973, U.S.A
}

\section{Abstract}

Beam-beam effects present one of major factors limiting the luminosity of colliders. In the linac-ring option of eRHIC design, an electron beam accelerated in a superconducting energy recovery linac collides with a proton beam circulating in the RHIC ring. There are some features of beam-beam effects, which require careful examination in linac-ring configuration. First, the beambeam interaction can induce specific head-tail type instability of the proton beam referred to as ' $k$ ink' instability. Thus, beam stability conditions should be established to avoid proton beam loss. Also, the electron beam transverse disruption by collisions has to be evaluated to ensure beam quality is good enough for the energy recovery pass. In addition, fluctuations of electron beam current and/or electron beam size, as well as transverse offset, can cause proton beam emittance growth. The tolerances for those factors should be determined and possible countermeasures should be developed to mitigate the emittance growth. In this paper, a soft Gaussian strong-strong simulation is used to study all of mentioned beam-beam interaction features and possible techniques to reduce the emittance growth.

\section{INTRODUCTION}

There is growing interest in physics community in building high energy, high luminosity polarized electronion collider in order to study the fundamental structure of nucleons and nuclei and details of QCD theory governing the interaction in nuclear matter. eRHIC will take advantage of existing RHIC ion collider at BNL with addition of an electron accelerator, which will generate high intensity electron beam.

The design of eRHIC based on an energy recovery linac opens a way to high luminosity of more than $10^{33} \mathrm{~cm}^{-2} \mathrm{~s}^{-}$ ${ }^{1}[1]$. In this so-called linac-ring design the electron accelerator includes a superconducting energy recover linac and several recirculating passes in order to accelerate the electrons to $10 \mathrm{GeV}$, with possible extension to higher energies. The fact that the electron beam collides with ions only once allows for higher strength of beam-beam interaction and, therefore, higher luminosity than could be achieved by using the electron storage ring. In Table 1, the basic parameters of present eRHIC linac-ring design are listed.

There are several features of beam-beam effect, special for the linac-ring design. In this paper we will focus on following effects: the proton beam instability of head-tail

\footnotetext{
*Work supported by U.S.DOE under contract No DE-AC02-98CH1886, DE-FG02-92ER40747 and NSF under contract No PHY-0552389 "yhao@ bnl.gov
}

type referred as 'kink instability', the electron beam disruption and proton emittance growth due to electron beam noise. All these effects have been studied using numerical simulations by specially written beam-beam simulation code. The results are presented in following sections.

Table 1. eRHIC parameter table

\begin{tabular}{|l|c|c|}
\hline & Proton beam & Electron beam \\
\hline Bunch Intensity & $2 . \mathrm{l} 1 \mathrm{1}$ & $1.2 \mathrm{e} 11$ \\
\hline Energy $(\mathrm{GeV})$ & 250 & 10 \\
\hline $\begin{array}{l}\text { RMS emittance } \\
(\mathrm{nm}-\mathrm{rad}), \mathrm{x} / \mathrm{y}\end{array}$ & $3.8 / 3.8$ & $1.0 / 1.0$ \\
\hline$\beta^{*}(\mathrm{~m}), \mathrm{x} / \mathrm{y}$ & $0.26 / 0.26$ & $1.0 / 1.0$ \\
\hline Bunch length $(\mathrm{cm})$ & 20 & 0.7 \\
\hline $\begin{array}{l}\text { Beam-beam } \\
\text { parameter, } \mathrm{x} / \mathrm{y}\end{array}$ & $0.015 / 0.015$ & $2.3 / 2.3$ \\
\hline $\begin{array}{l}\text { Disruption parameter, } \\
\mathrm{x} / \mathrm{y}\end{array}$ & $1.3 \mathrm{e}-3 / 1.3 \mathrm{e}-3$ & $5.9 / 5.9$ \\
\hline
\end{tabular}

\section{KINK INSTABLITY}

The periodic beam-beam interaction between proton and electron beams may excite the strong head-tail type instability, referred as the kink instability, on the proton beam. Previous works [2,3] analytically considered the instability using linear approximation of beam-beam force.

We use soft Gaussian strong-strong simulation code to study the kink instability in the eRHIC linac-ring scheme. Proton bunches are divided in several longitudinal slices and the electro-magnetic field is calculated for every slice using its transverse size and its beam intensity. The electron beam, whose bunch length is much shorter than that of protons, is considered as an infinitely thin slice. Such approach allows exploring relevant physics phenomena and requires much less calculation time than the particle-in-cell method. In our simulation, both full nonlinear beam-beam force (1) and only its linear part have been used. The variation of beta functions along the interaction region, hourglass effect, has been also taken into account.

$$
F_{r}=\frac{n e^{2}}{2 \pi \varepsilon_{0} r}\left[1-\exp \left(-\frac{r^{2}}{2 \sigma^{2}}\right)\right]
$$

Figure 1 shows that the example of fast emittance growth which occurs at zero chromaticity, with the beambeam force set to be nonlinear and included hourglass effect. The growth rate decreases as the proton intensity drops down. At the intensity of $1 . e 10$ protons per bunch, the proton beam is below threshold with no fast emittance growth. 


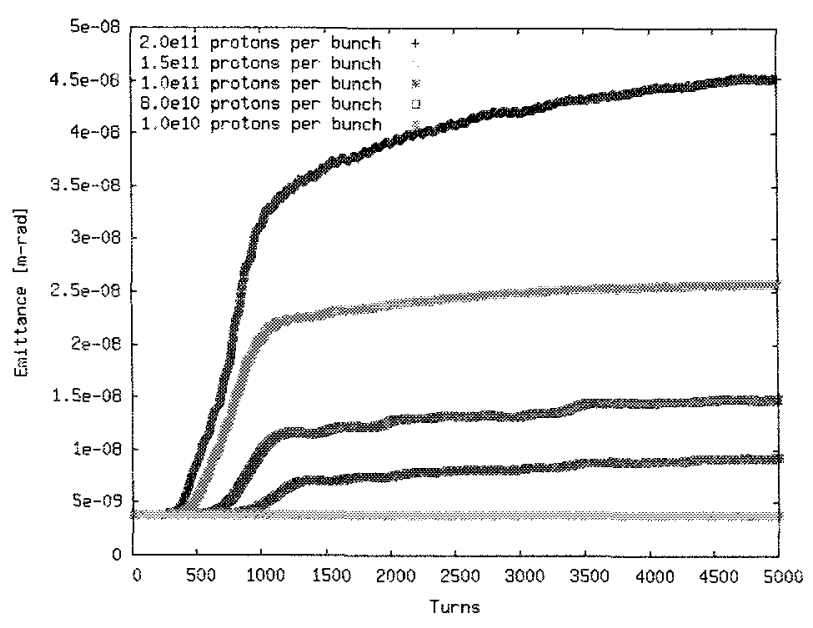

Figure 1: Emittance growth vs. turns

Frequency analysis done in linear beam-beam force approximation, zero chromaticity and without the hourglass effect (Figure 2) allows finding the threshold of strong head-tail effect. The mode merging happens at the proton intensity of $1.6 \mathrm{e} 10$ particles per bunch, much lower than the design parameter $2 \mathrm{e} 11$ protons per bunch. On the turns when fast emittance growth is seen, the 0 and 1 collective modes are well seen in the dipole moment pattern, as shown in Figure 3. Here we use RHIC synchrotron tune 0.0043 in simulation.

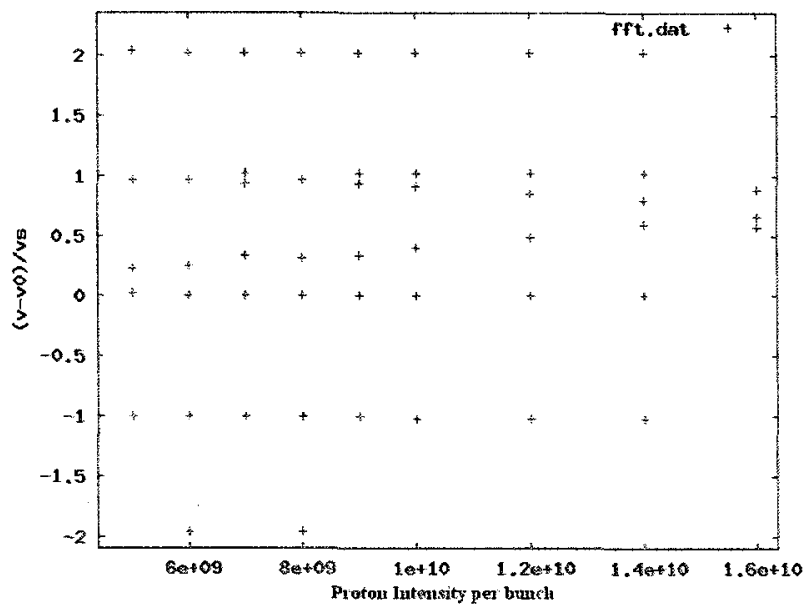

Figure 2: Frequency of collective modes vs. proton intensity

Landau damping can be introduced to suppress the instability. For eRHIC design parameters, we found that large enough chromaticity and nonlinear beam-beam force can stabilize the beam by suppressing the fast emittance growth. As shown in Figure 4, for nonlinear beam-beam force case, positive chromaticity of 4-5 units minimizes the emittance growth ratio after 3000 turns. The energy spread of proton beam used in simulation is 0.001 .

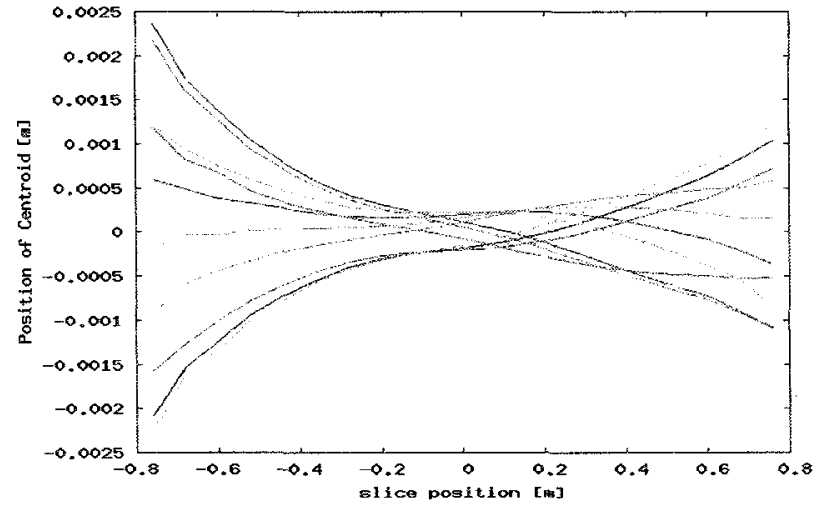

Figure 3: Turn by turn (900-910 turn) transverse centroid vs. longitudinal position, linear force approximation without hourglass effect.

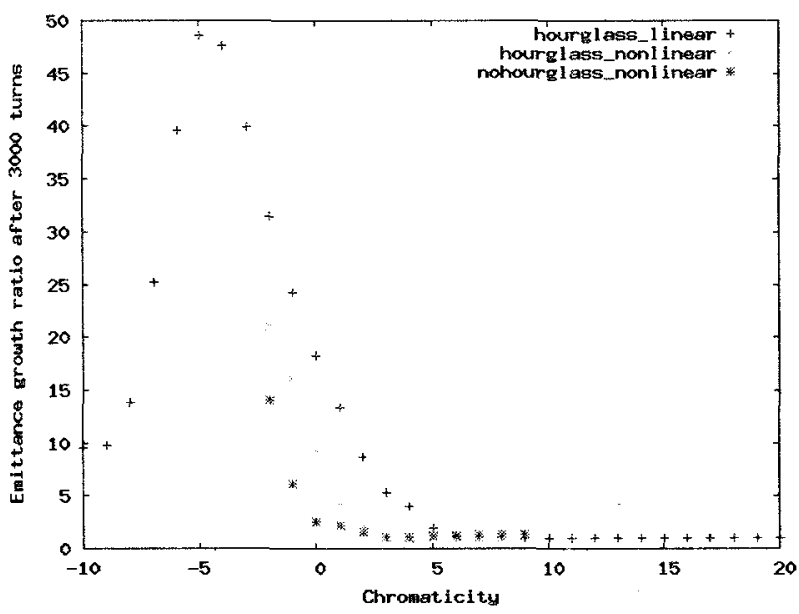

Figure 4: Emittance growth factor vs. chromaticity

\section{NOISE EFFECT IN ELECTRON BUNCH}

On each turn a proton bunch collides with a new electron bunch from the ERL accelerator. The parameters of electron beam; such as bunch intensity and transverse beam size may vary from turn to turn. This leads to the fluctuations of the proton beam-beam parameter that result in the proton beam size increasing slowly driven by the noisy beam-beam interactions.

In our simplified analytical approach, we treat the beam-beam interaction as a thing focusing lens with the strength given by

$$
d=1 / f=\frac{4 \pi \xi}{\beta^{*}}
$$

where $\beta^{*}$ is the beta function at IP, $\xi$ is the beam-beam parameter.

Solving the equation of linear transverse motion with added fluctuation $\delta \mathrm{d}$ of beam-beam quadrupole, one obtains the average growth rate of the mean square beam size as::

$$
\frac{d\left\langle x^{2}\right\rangle}{d t}=\frac{\beta^{* 2} d\left\langle x^{2}\right\rangle}{2 T} \frac{\left\langle(\delta d)^{2}\right\rangle}{d^{2}}
$$


with $\mathrm{T}$ as the revolution period.

From Eq (3), with existence of noise in electron beam, the proton beam size will exponentially grow with respect to time. The growth time is

$$
\tau_{\langle x\rangle}=\frac{4 T}{\beta^{* 2} d^{2}} \frac{d^{2}}{\left\langle(\delta d)^{2}\right\rangle}=\frac{T}{4 \pi^{2} \xi^{2}} \frac{d^{2}}{\left\langle(\delta d)^{2}\right\rangle}
$$

Figure 5 demonstrates the relation between the growth time and the fluctuation of proton beam-beam parameter caused by different electron bunch intensity and beam size from shot to shot. For acceptable beam size growth time, the fluctuations of beam-beam focusing strength should be not larger than 5.e-4.

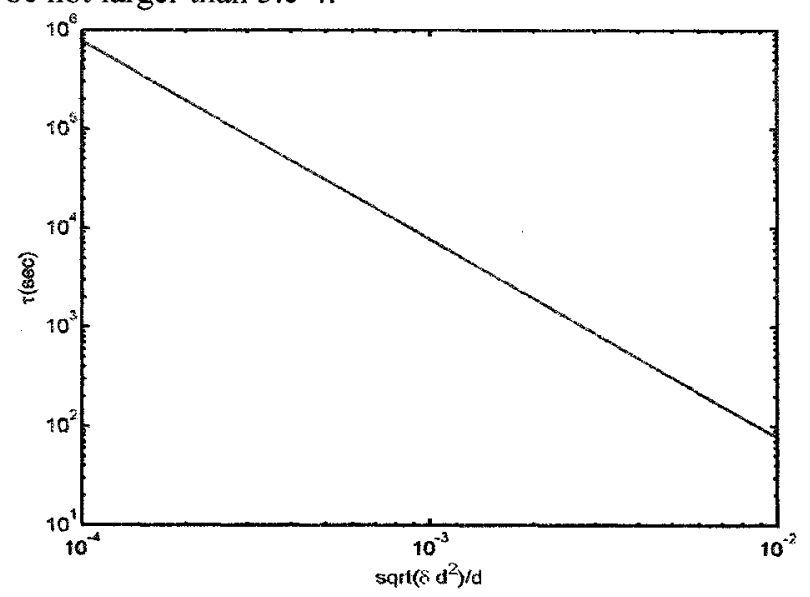

Figure 5: Beam size growth time vs. fluctuations of beambeam strength

\section{ELECTRON BEAM DISRUPTION}

The expected value of disruption parameter of electron beam can reach 5.9. The nonlinear part of the beam-beam force causes considerable electron emittance growth. In the same time, the linear focusing due to the beam-beam force provides the electrons with extra phase advance and, therefore, creates a mismatch between the design lattice optics and the electron transverse distribution.

Simulations were done assuming protons to have Gaussian transverse and longitudinal distributions and the beam-beam force described by the expression (1). The simulations show how electron RMS emittance and beam size evolve due to the interaction with the protons throughout the collision region (Figure 6).

Disrupted electron beam goes through recirculating passes during its deceleration. The magnet aperture should be large enough to avoid excessive beam loss. Figure 7 presents the beam loss power dependence on the magnet aperture for two types of initial electron beam distribution (Gaussian (denoted as GS in Figure 7) and Beer-Can (denoted as $\mathrm{BC})$ ) and for two electron energies ( $3 \mathrm{GeV}$ and $20 \mathrm{GeV}$ ). For the worst energy case of $3 \mathrm{GeV}$ beam the least magnet aperture can be as small as $3.5 \mathrm{~mm}$ with the Beer-Can beam distribution.

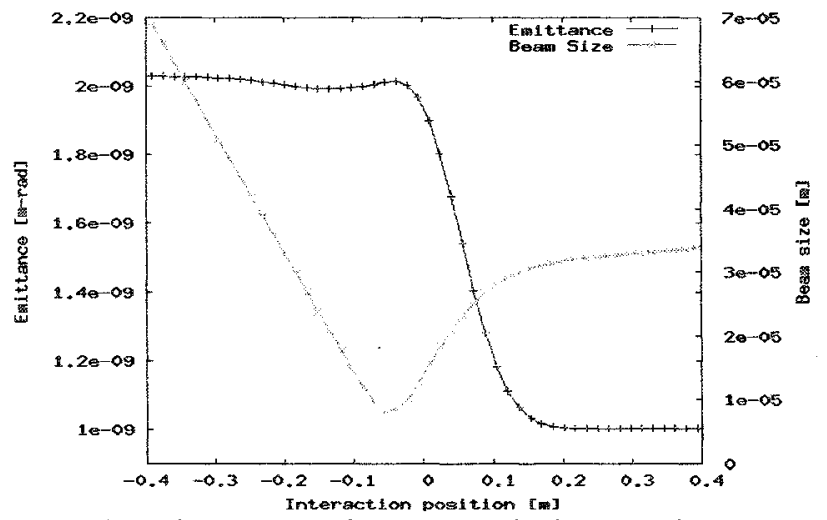

Figure 6: Electron emittance and beam size vs. longitudinal position. Positive longitudinal position corresponds to the head of the proton bunch.

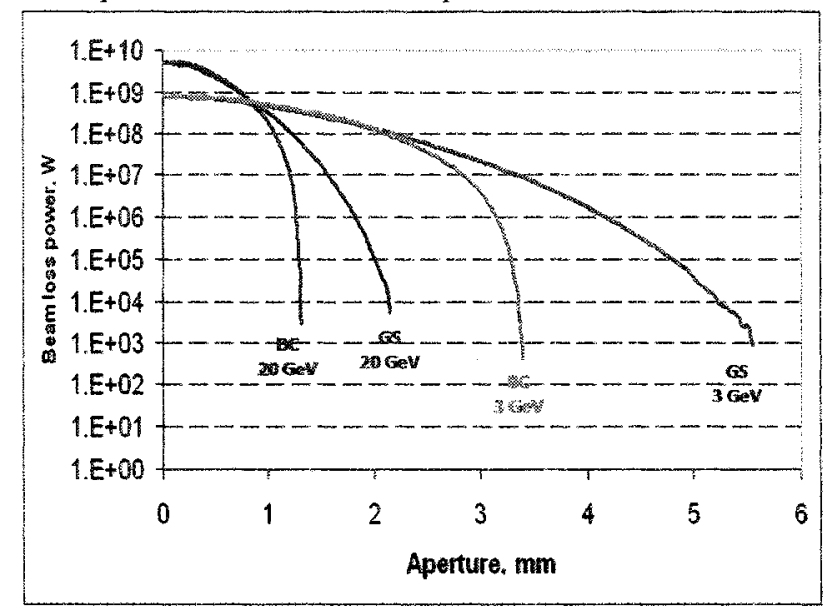

Figure 7: Beam loss power vs. magnet aperture

\section{CONCLUSION}

Three aspects of beam-beam interactions for the ERLbased eRHIC design have been considered. It was found that the design proton beam intensity is above kink instability threshold. However, the nonlinear beam-beam field and large enough chromaticity stabilize the beam and eliminate the emittance growth. To reduce the proton beam size growth to the acceptable level, the fluctuation of electron beam parameters, such as the emittance and bunch intensity should be not larger than 5e-04. The electron beam disruption doubles the rms emittance of electron beam and seriously distorts electron optics. The size of the disrupted electron beam has been used to define the minimal magnet aperture which can be as small as $3.5 \mathrm{~mm}$ for the case of Beer-Can distribution.

\section{REFERENCES}

[1] eRHIC ZDR, C-AD/AP 142 (2004).

[2] E.A.Perevedentsev and A.A.Valishev, Phys. Rev. STAB, v. 4, 024403 (2001).

[3] R.Li, et al, Proceedings of 2001 Particle Accelerator Conference, Chicago. 